\title{
LOWER AND UPPER BOUNDS OF SHORTEST PATHS IN REACHABILITY GRAPHS
}

\author{
P. K. MISHRA \\ Received 22 March 2004
}

\begin{abstract}
We prove the following property for safe marked graphs, safe conflict-free Petri nets, and live and safe extended free-choice Petri nets. We prove the following three results. If the Petri net is a marked graph, then the length of the shortest path is at most $(|T|-1) \cdot|T| / 2$. If the Petri net is conflict free, then the length of the shortest path is at most $(|T|+1) \cdot|T| / 2$. If the Petri net is live and extended free choice, then the length of the shortest path is at most $|T| \cdot|T+1| \cdot|T+2| / 6$, where $T$ is the set of transitions of the net.
\end{abstract}

2000 Mathematics Subject Classification: 68R10, 90C35, 94C15.

1. Introduction. Let $M_{1}, M_{2}$ be the two markings of the reachability graph of a safe Petri net such that $M_{2}$ is reachable from $M_{1}$. Since a safe Petri net with $n$ places has at most $2^{n}$ markings, this length is less than $2^{n}$. However, in some situations, we would like to have a better bound. An example is a system with some state-a state reachable from any other reachable state-which should be reached after a recovery action. If the home state can be only reached after an exponential number of actions, then the system cannot recover in reasonable time.

Another reason to study this question is that the length of shortest paths between pairs of markings is related to the complexity of the model checker for arbitrary safe Petri nets.

If the Petri net is a marked graph, then the length of the shortest path is at most

$$
\frac{(|T|-1) \cdot|T|}{2}
$$

If the Petri net is conflict free, then the length of the shortest path is at most

$$
\frac{(|T|+1) \cdot|T|}{2}
$$

If the Petri net is live and extended free-choice, then the length of the shortest path is at most

$$
\frac{|T| \cdot|T+1| \cdot|T+2|}{6},
$$

where $T$ is the set of transitions of the net.

The paper is organized as follows. Section 2 contains basic definitions and results. Section 3 studies so-called biased sequences. Using the results of Section 3, our three 
results are proved in Sections 4 and 5. Finally, Section 6 shows that for safe persistent systems there exist no polynomial bounds for the lengths of shortest paths.

2. Preliminaries. Let $S$ and $T$ be finite and nonempty disjoint sets and let

$$
F \subseteq(S \times T) \cup(T \times S)
$$

If for each $x \in S$ and $y \in T$ it happens that $(x, y) \in F$ or $(y, x) \in F$, then $N=(S, T, F)$ is called a net. $S$ is the set of places and $T$ is the set of transitions of $N$. Pre- and postsets of elements are denoted by the dot notation

$$
\begin{aligned}
& \bullet x=\{y \mid(y, x) \in F\}, \\
& x \bullet=\{y \mid(x, y) \in F\} .
\end{aligned}
$$

This notion is extended to the set of elements also. A set $c$ of transitions of $N$ is a conflict set if either $c=s \bullet$ for some places $s$ or $c=\{t\}$ for some transition $t$ satisfying $\bullet t=\phi$.

A marking of $N$ is a mapping $M: S \rightarrow \mathbb{N}^{+}$, where $\mathbb{N}^{+}$is the set of nonnegative integers. A place $s$ is called marked by a marking $M$ if $M(s)>0$. A marking $M$ enables a transition $t$ if it marks every place of $\bullet t$. The occurrence of an enabled transition $t$ leads to the successor marking $M^{1}$ (written $M \rightarrow M^{1}$ ) which is defined for every place of $s$ by

$$
M^{1}(s)= \begin{cases}M(s)-1 & \text { if } s \in \bullet^{\bullet} t t^{\bullet}, \\ M(s)+1 & \text { if } s \in t^{\bullet} / t^{\prime}, \\ M(s) & \text { if } s \notin \bullet^{\bullet} t t^{\bullet} \text { or } s \in{ }^{\bullet} t \cap t^{\bullet} .\end{cases}
$$

If $M_{0} \stackrel{t_{1}}{\longrightarrow} M_{1} \stackrel{t_{2}}{\longrightarrow} \cdots \stackrel{t_{n}}{\longrightarrow} M_{n}$, then $\sigma=t_{1} t_{2} \cdots t_{n}$ is called an occurrence sequence and we write $M_{0} \stackrel{\sigma}{\longrightarrow} M_{n}$. This notion includes the empty sequence $\varepsilon: M \stackrel{\varepsilon}{\longrightarrow} M$ for each marking $M$.

A sequence $\sigma$ is enabled at a marking $M$ if $M \stackrel{\sigma}{\longrightarrow} M^{1}$ for some marking $M^{1}$. We call $M^{1}$ reachable from $M$ if $M \stackrel{\sigma}{\longrightarrow} M^{1}$ for some occurrence sequence $\sigma$. The set of all markings reachable from $M$ is denoted by $[M\rangle$. Given a sequence $\sigma$ of transitions and a transition $t, \#(t, \sigma)$ denotes the number of occurrences of $t$ in $\sigma$. For a set of

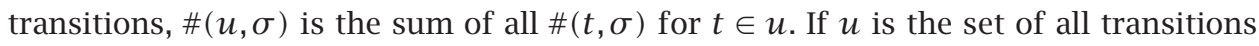
of the net, then $\#(u, \sigma)$ is called the length of $\sigma$.

A sequence $\sigma$ of transitions is a permutation of a sequence $\tau$ if $\#(t, \sigma)=\#(t, \tau)$ for every transition $t$. A net system is a pair $\left(N, M_{0}\right)$ where $N$ is a net and $M_{0}$ is a marking of $N$, called initial marking of $\left(N, M_{0}\right)$. A marking is called reachable in a system $\left(N, M_{0}\right)$ if it is reachable from $M_{0}$. A system $\left(N, M_{0}\right)$ is called live if for every reachable marking $M$ and every transition $t$ there exists a marking $M^{1} \in[M\rangle$ that enables $t$. It is called safe if every reachable marking $M$ satisfies $M(s) \leq 1$ for every place $s$. The reachability graph $(V, E)$ of a system $\left(N, M_{0}\right)$ is the directed graph defined by $V=\left[M_{0}\right\rangle$ and $E=\left\{\left(M_{1}, M_{2}\right) \in V \times V \mid M_{1} \stackrel{t}{\longrightarrow} M_{2}\right.$ for some transitions $\left.t\right\}$.

We use the following two results, which follow immediately from the occurrence rule and are well known. 
LEMMA 2.1. (i) Let $M_{1} \stackrel{\sigma}{\longrightarrow} M_{2}$ be an occurrence sequence. Then, for every place $s$,

$$
M_{2}(s)=M_{1}(s)+\#(\bullet s, \sigma)-\#(s \bullet, \sigma) .
$$

(ii) Let $M_{1} \stackrel{\sigma}{\longrightarrow} M_{2}$ and let $M_{1} \stackrel{\tau}{\longrightarrow} M_{3}$ be an occurrence sequence. If $\tau$ is a permutation of $\sigma$, then $M_{2}=M_{3}$.

3. Biased occurrence sequences. The purpose of this section is to prove Theorem 3.4, which yields an upper bound for the shortest paths between two markings $M_{1}$ and $M_{2}$, when $M_{2}$ can be reached from $M_{1}$ by means of a so-called biased occurrence sequence. This theorem will easily lead to our first two results concerning marked graphs and conflict-free systems. Moreover, it will be used as a lemma in the proof of our third result on extended free-choice systems.

DEFINITION 3.1. A sequence $\sigma$ of transitions of a net $N$ is called biased if, for every conflict set $c$ of $N$, at most one transition of $c$ occurs in $\sigma$.

LEMMA 3.2. Let $M_{1} \stackrel{\sigma}{\rightarrow} M_{2}$ be a biased occurrence sequence of a net. If $\sigma=\sigma_{1} \sigma_{2} t$ such that

(i) $t$ is a transition that does not occur in $\sigma_{1}$,

(ii) every transition occurring in $\sigma_{2}$ also occurs in $\sigma_{1}$,

then $M_{1} \stackrel{\sigma_{1} t \sigma_{2}}{\longrightarrow} M_{2}$ is also an occurrence sequence.

Proof. By induction on the length of $\sigma_{2}$.

BASE. If $\sigma_{2}=\varepsilon$, then $\sigma_{1} \sigma_{2} t=\sigma_{1} t=\sigma_{1} t \sigma_{2}$.

STEP. If $\sigma_{2} \neq \varepsilon$, then $\sigma_{2} t=\sigma_{2}^{1} u$ for some sequence $\sigma_{2}$ and some transition $u$.

Let $M_{1} \stackrel{\sigma_{1}}{\longrightarrow} M_{3} \stackrel{\sigma_{2}}{\longrightarrow} M_{4} \stackrel{u}{\longrightarrow} M_{5} \stackrel{t}{\longrightarrow} M_{2}$.

We first prove $M_{4} \stackrel{t}{\longrightarrow} M_{6} \stackrel{u}{\longrightarrow} M_{2}$ for some marking $M_{6}$. If $t=u$, we are done; so assume $t \neq u$.

We claim that $M_{4}$ enables $t$. Let $s$ be an arbitrary place in the preset of $t$; we prove that $M_{4}(s)>0$. Consider the following two cases.

(i) If $s \notin u \bullet$, then $M_{4}(s) \geq M_{5}(s)$. Since $t$ is enabled at $M_{5}$, we have $M_{5}(s)>0$.

(ii) If $s \in u \bullet$, we have

$$
M_{5}(s)=M_{1}(s)+\#\left(\bullet s, \sigma_{1} \sigma_{2}\right)-\#\left(s \bullet, \sigma_{1} \sigma_{2}\right) .
$$

By Lemma 3.2(ii) and since $u$ occurs in $\sigma_{2}, u$ occurs at least twice in $\sigma_{1} \sigma_{2}$. Since $u \in \bullet s$, we get $\#\left(\bullet s, \sigma_{1} \sigma_{2}\right) \geq 2$.

Since $\sigma$ is biased and $t$ occurs in $\sigma, t$ is the only transition in the postset of $s$ that

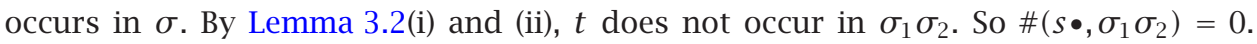
Therefore $M_{5}(s) \geq 2$. Since $M \stackrel{u}{\longrightarrow} M_{5}$, we get $M_{4}(s) \geq 1$. Since $M_{4}$ enables $t, M_{4} \stackrel{t}{\longrightarrow} M_{6}$ for some marking $M_{6}$. Since $\sigma$ is biased,

$$
\bullet u \cap \bullet t=\phi
$$

so the occurrence of $t$ does not disable $u$, and hence $u$ is enabled at $M_{6}$. Since $u t$ and $t u$ are permutations of each other, we finally get $M_{4} \stackrel{t}{\longrightarrow} M_{6} \stackrel{u}{\longrightarrow} M_{2}$. 
The application of the induction hypothesis to $\sigma_{1} \sigma_{2}^{1} t$ (taking $\sigma_{2}^{1}$ for $\sigma_{2}$ ) yields an occurrence sequence $M_{4} \stackrel{\sigma_{1} t \sigma_{2}^{1}}{\longrightarrow} M_{6}$. The result follows because $M_{6} \stackrel{u}{\longrightarrow} M_{2}$ and $\sigma_{2}^{1} u=$ $\sigma_{2}$.

LEMMA 3.3. Let $M_{1} \stackrel{\sigma}{\longrightarrow} M_{2}$ be a biased occurrence sequence of a net. There exists a permutation $\sigma_{1} \sigma_{2}$ of $\sigma$ such that $M_{1} \stackrel{\sigma_{1} \sigma 2}{\longrightarrow} M_{2}$; no transition occurs more than once in $\sigma_{1}$ and every transition occurring in $\sigma_{2}$ also occurs in $\sigma_{1}$.

Proof. By induction on the length of $\sigma$.

BASE. If $\sigma=\varepsilon$, take $\sigma_{1}=\sigma_{2}=\varepsilon$.

STEP. If $\sigma \neq \varepsilon$, then $\sigma=\tau t$ for some sequence $\tau$ and some transition $t$.

By the induction hypothesis, there is a permutation $\tau_{1} \tau_{2}$ of $\tau$, enabled at $M_{1}$, such that no transition occurs more than once in $\tau_{1}$ and every transition in $\tau_{2}$ also occurs in $\tau_{1}$.

If $t$ occurs in $\tau_{1}$, then $\sigma_{1}=\tau_{1}$ and $\sigma_{2}=\tau_{2} t$ satisfy the requirements.

If $t$ does not occur in $\tau_{1}$, then $\tau_{1} \tau_{2} t$ satisfies the conditions of Lemma 3.2, and so $M_{1} \stackrel{\tau_{1} t \tau_{2}}{\longrightarrow} M_{2}$ is an occurrence sequence. Take $\sigma_{1}=\tau_{1} t$ and $\sigma_{2}=\tau_{2}$.

THEOREM 3.4. Let $M_{1}$ be a reachable marking of a safe system and let $M_{1} \stackrel{\sigma}{\longrightarrow} M_{2}$ be a biased occurrence sequence. Let $k$ be the number of distinct transitions occurring in $\sigma$. There exists an occurrence sequence $M_{1} \stackrel{\tau}{\longrightarrow} M_{2}$ such that the length of $\tau$ is at most $k \cdot(k+1) / 2$.

Proof. By induction on the length of $\sigma$.

BASE. For $\sigma \neq \varepsilon$, choose $\tau=\varepsilon$.

STEP. If $\sigma=\varepsilon$, then by Lemma 3.3 there exists a permutation $\tau_{1} \boldsymbol{T}_{2}$ of $\sigma$ such that $M_{1} \stackrel{\tau_{1} \tau_{2}}{\longrightarrow} M_{2}$; every transition occurring in $\tau_{2}$ occurs in $\tau_{1}$ and no transition occurs in $\tau_{1}$ more than once. Since $\sigma$ is not the empty sequence, $\tau_{1}$ is not empty, and therefore $\tau_{2}$ is shorter than $\sigma$. Let $M_{1} \stackrel{\tau_{1}}{\longrightarrow} M_{3} \stackrel{\tau_{2}}{\longrightarrow} M_{2}$.

We distinguish two cases.

(i) Every transition occurring in $\tau_{1}$ occurs in $\tau_{2}$. Again by Lemma 3.3 there are a permutation $p_{1}$ and a permutation $p_{2}$ of $\tau_{2}$ such that $M_{3} \stackrel{p_{1} p_{2}}{\longrightarrow} M_{2}$ with every transition occurring in $p_{2}$ and $p_{1}$, and no transition occurs in $p_{1}$ more than once. Then a transition occurs in $\tau_{1}$ if and only if it occurs in $p_{1}$. Moreover, no transition occurs more than once in either sequence. So every transition $t$ satisfies $\#\left(t, \tau_{1}\right)=\#\left(t, p_{1}\right)$, that is, $\tau_{1}$ and $p_{1}$ are permutations of each other. Let $M_{1} \stackrel{\tau_{1}}{\longrightarrow} M_{3} \stackrel{p_{1}}{\longrightarrow} M_{4}$, then for each place it holds that

$$
M_{4}(s)=M_{1}(s)+\#\left(\bullet s, \tau_{1}\right)-\#\left(s, \tau_{1}\right)+\#\left(\bullet s, p_{1}\right)-\#\left(s \bullet, p_{1}\right)
$$

and hence

$$
M_{4}(s)=M_{1}(s)+2\left(\#\left(\bullet s, \tau_{1}\right)-\#\left(s \bullet, \tau_{1}\right)\right) .
$$

Thus, since $M_{1}, M_{4} \in\left[M_{0}\right\rangle$ and $\left(N, M_{0}\right)$ is safe, we get

$$
\#\left(\bullet s, \tau_{1}\right)-\#\left(s \bullet, \tau_{1}\right)=0 .
$$


So

$$
M_{3}(s)=M_{1}(s)+\#\left(\bullet s, \tau_{1}\right)-\#\left(s \bullet, \tau_{1}\right)=M_{1}(s) .
$$

Therefore,

$$
M_{1}=M_{3}, \quad M_{1} \rightarrow M_{2}
$$

Since $\tau_{2}$ is shorter than $\sigma$, we can apply the induction hypothesis to it, which yields an occurrence sequence $M_{1} \stackrel{\tau}{\longrightarrow} M_{2}$ of the desired length.

(ii) There exists a transition which occurs in $\tau$. We apply the induction hypothesis to $M_{3} \stackrel{\tau_{2}}{\longrightarrow} M_{2}$. Since the number of distinct transitions occurring in $\tau_{2}$ is at most $k-1$, we get a sequence $M_{3} \stackrel{p}{\longrightarrow} M_{2}$ such that the length of $p$ is at most $k \cdot(k-1) / 2$. Since no transition occurs in $\tau_{1}$ more than once, its length is just $k$. Let

$$
\tau=\tau_{1} p
$$

We have $M_{1} \stackrel{\tau}{\longrightarrow} M_{2}$. Moreover, the length of $\tau$ is at most

$$
k+\frac{k \cdot(k-1)}{2}=\frac{k \cdot(k+1)}{2} .
$$

4. T-systems, marked graphs, and conflict-free systems. If a system has no forward branching places (i.e., $|s \bullet| \leq 1$ for every place), then all its occurrence sequences are biased, so Theorem 3.4 applies to every occurrence sequence and we get the following result.

THeORem 4.1. Let $\left(N, M_{0}\right)$ be a safe system where $N=(S, T, F)$ and $|s \bullet| \leq 1$ for every $s \in S$, and let $M_{1}$ be a reachable marking. Let $M_{2}$ be a marking reachable from $M_{1}$. There exists an occurrence sequence $M_{1} \stackrel{\tau}{\longrightarrow} M_{2}$ such that the length of $\tau$ is at most $(|T|+1) \cdot|T| / 2$.

Proof. Since $M_{2}$ is a reachable marking from $M_{1}$, there exists an occurrence sequence $M_{1} \stackrel{\sigma}{\longrightarrow} M_{2}$. $\sigma$ is biased because every conflict set of $N$ contains exactly one transition, and the number of distinct transitions occurring in $\sigma$ is at most $|T|$ and, using the Theorem 3.4, the result is obvious.

4.1. $T$-systems. Theorem 4.1 applies in particular to $T$-systems, in which $|s \bullet| \leq 1$ and, moreover, $|\bullet s| \leq 1$ for every place $s$. The bound of the theorem is reachable for $T$-systems, that is, there exist a $T$-system and pairs of reachable markings $M_{1}, M_{2}$ for which the bound is the exact value of the length of the shortest path leading from $M_{1}$ to $M_{2}$.

4.2. Marked graphs. $T$-systems satisfying $|\bullet s|=1=|s \bullet|$ for every place $s$ are called marked graphs in [6] or synchronization graphs in [10]. For this class of systems, we can obtain a stronger result.

THEOREM 4.2. Let $\left(N, M_{0}\right)$ be a safe system where $N=(S, T, F)$ and $|\bullet s|=1=|s \bullet|$ for everys $\in S$. Let $M_{1}$ be a reachable marking. Let $M_{2}$ be a marking reachable from $M_{1}$. 
There exists an occurrence sequence $M_{1} \stackrel{\tau}{\longrightarrow} M_{2}$ such that the length of $\tau$ is at most $(|T|-1) \cdot|T| / 2$.

Proof. Since $M_{2}$ is a reachable marking from $M_{1}$, there exists an occurrence sequence $M_{1} \stackrel{\sigma}{\longrightarrow} M_{2}$. We can further assume that $\sigma$ has minimal length. We claim that at least one transition of $N$ does not occur in $\sigma$. The result then follows from Theorem 3.4 taking $k=|T|-1$.

Assume that every transition of $N$ occurs in $\sigma$. By Lemma 3.3, there exists a permutation of $\sigma$ such that $M_{1} \stackrel{\sigma_{1} \sigma_{2}}{\longrightarrow} M_{2}$, no transition occurs more than once in $\sigma_{1}$, and every transition occurring in $\sigma_{2}$ also occurs in $\sigma_{1}$. Therefore every transition of $N$ occurs in $\sigma$ and, moreover, they occur exactly once. Let $M_{3}$ be the marking such that $M_{1} \stackrel{\sigma_{1}}{\longrightarrow} M_{3}$. We claim that $M_{1}=M_{3}$. Let $s$ be an arbitrary place of $N$; by Lemma 2.1 we have

$$
M_{3}(s)=M_{1}(s)+\#\left(\bullet s, \sigma_{1}\right)-\#\left(s \bullet, \sigma_{1}\right) .
$$

Since $s$ has exactly one input and one output place, we get

$$
\#\left(\bullet s, \sigma_{1}\right)=1=\#\left(s \bullet, \sigma_{1}\right) .
$$

So $M_{3}(s)=M_{1}(s)$, which proves the claim. Since $M_{3}=M_{1}$, we have $M_{1} \stackrel{\sigma_{2}}{\longrightarrow} M_{2}$. Since $\sigma_{1}$ is nonempty, $\sigma_{2}$ is shorter than $\sigma$, which contradicts the minimality of $\sigma$.

Like the bound of $T$-systems, the bound of Theorem 4.2 is also tight. Consider the family of systems. Adding a transition $t_{n+1}$ such that

$$
\bullet t_{n+1}=\left\{s_{2 n}\right\}, \quad t \bullet_{n+1}=\left\{s_{2 n-1}\right\}
$$

yields a marked graph with $|T|=n+1$ transitions. The transition $t_{n+1}$ does not occur in the shortest path from $M_{\text {odd }}$ to $M_{\text {even }}$. As shown before, the shortest path from $M_{\text {odd }}$ to $M_{\text {even }}$ needs

$$
\frac{n \cdot(n+1)}{2}=\frac{(|T|-1) \cdot|T|}{2}
$$

transition occurrences.

4.3. Conflict-free systems. Theorem 4.1 can also be generalized to conflict-free nets, a well-known class of nets studied in [16].

DEFINITION 4.3. A net $N$ is called conflict-free if every place $s$ of $N$ satisfies either $|s \bullet| \leq 1$ or $s \bullet \subseteq \bullet$. A system $\left(N, M_{0}\right)$ is conflict-free if $N$ is conflict-free.

THEOREM 4.4. Let $\left(N, M_{0}\right)$ be a safe conflict-free system, where $N=(S, T, F)$ and $M_{1}$ is a reachable marking. Let $M_{2}$ be a marking reachable from $M_{1}$. There exists an occurrence sequence $M_{1} \stackrel{\tau}{\longrightarrow} M_{2}$ such that the length of $\tau$ is at most $(|T|+1) \cdot|T| / 2$.

Proof. Let $R$ be the set of places of $N$ with more than one transition in their postset. We proceed by induction on $|R|$.

BASE. For $R=\phi$, the result follows by Theorem 4.1. 
STEP. For $R \neq \phi$, let $s \in R$ be an element.

Using the definition of conflict-free systems [3, 12, 13] and by induction hypothesis, the result is obvious.

5. Extended free-choice systems. In this section, we obtain an upper bound for the length of the shortest paths between two reachable markings of a live and safe extended free-choice system. It is never greater than $|T| \cdot|T+1| \cdot|T+2| / 6$, where $T$ is the set of transitions of the net. Extended free-choice systems are a generalization of a free-choice system introduced in [11].

Definition 5.1. A net $N$ is extended free-choice if every two places $s, s^{1}$ of $N$ satisfy either $s \bullet=s^{1} \bullet$ or $s \bullet \cap s^{1} \bullet=\phi$; that is, if its conflict sets constitute a partition of its set of transitions. A system $\left(N, M_{0}\right)$ is extended conflict-free if $N$ is extended conflict-free. Note that every net without forward branching places is extended free-choice [5]. The proof of our result is based on the notion of conflict order.

DEFINITION 5.2. Let $N$ be an extended free-choice net and let $T$ be the set of transitions of $N$. A conflict order $\leq \subseteq T \times T$ is a partial order such that two transitions $t$ and $u$ are comparable (i.e., $t \leq u$ or $u \leq t$ ) if and only if they belong to the same conflict set. For elements $u, t \in T$, the expression $u<t$ denotes $u \leq t$ and $u \neq t$. Let $\sigma$ be a sequence of transitions of $N$. A conflict order $\leq$ is said to agree with $\sigma$ if, for every conflict set $c$, either no transition of $c$ occurs in $\sigma$ or the last transition of $c$ occurring in $\sigma$ is maximal, that is, the greatest transition of $c$ with respect to $\leq$.

We can now define, given an occurrence sequence $\sigma$ and a conflict order $\leq$, the set of permutations of $\sigma$ which are ordered with respect to $\leq$.

Definition 5.3. Let $N$ be an extended free-choice net and let $\leq$ be a conflict order. A sequence $\tau$ is called sorted with respect to $\leq$ (or $\leq$-sorted) if every two transitions $t, u$ satisfy $t<u$ and $t$ does not occur after $u$ in $\tau$.

Prefixes of sorted permutations of given sequence $\sigma$ will play a particular important role in the sequel $[8,17]$. We will need the following lemma.

LEMMA 5.4. Let $\sigma$ be a sequence of transitions of an extended free-choice net and let $\leq$ be a conflict order. Let $\tau$ be a prefix of a s-sorted permutation of $\sigma$. Let $t$ be $a$ transition satisfying

(i) $\#(t, \tau)<\#(t, \sigma)$,

(ii) $\#(u, \tau)=\#(u, \sigma)$ for every transition $u$ satisfying $u<t$.

Then, the sequence $\tau t$ is also a prefix of a $\leq$-sorted permutation of $\sigma$.

Proof. Since $\tau$ is a prefix of a $\leq$-sorted permutation of $\sigma$, there exists a sequence $p$ such that $\tau p$ is a $\leq$-sorted permutation of $\sigma$. By Lemma 5.4(i), $t$ occurs in $p$. Let $p^{\prime}$ be the sequence obtained from $p$ by deletion of the first occurrence of $t$ in $p$. Then the sequence $\tau t p^{\prime}$ is again a permutation of $\sigma$. It is sorted because by Lemma 5.4(ii), no transition $u$ satisfying $u<t$ occurs in $p$ and $p^{\prime}$.

We outline the proof of the result. Let $\left(N, M_{0}\right)$ be a live and safe extended free choice system, let $M_{1}$ be a reachable marking, and let $M_{1} \stackrel{\sigma}{\longrightarrow} M_{2}$ be an occurrence sequence. We show that 
(i) there exist a conflict order $\leq$ that agrees with $\sigma$ and a $\leq$-sorted permutation $\tau$ of $\sigma$ such that

$$
M_{1} \stackrel{\tau}{\longrightarrow} M_{2}
$$

(ii) $\tau=\tau_{1}, \tau_{2}, \ldots, \tau_{k}$, where $\tau_{i}$ is a biased sequence for every $i$, and $k$ is less than or equal to the number of transitions of $N$.

Using (ii) and Theorem 3.4, we prove that there exists a sequence $p_{1}, p_{2}, \ldots, p_{k}$ of bounded length. Of these two steps, (i) is more involved; (ii) follows easily from the definition of $\leq$-sorted permutation. To prove (i), we make use of the well-known decomposition theorem of the theory of free-choice system. We recall both the definition of $S$-component and the decomposition theorem.

Definition 5.5. An $S$-net is a net which does not satisfy $|\bullet t|=|t \bullet|=1$ for each transition $t$. A system $\left(N, M_{0}\right)$ is an $S$-system if $N$ is an $S$-net [9].

DEFINITION 5.6. A strongly connected $S$-net, $N_{1}$, is an $S$-component of a net $N$ if for every place $s$ of $N_{1}$ the following hold:

(1) $s$ is a place of $N$;

(2) the preset of $s$ in $N_{1}$ equals the preset of $s$ in $N$;

(3) the postset of $s$ in $N_{1}$ equals the postset of $s$ in $N$.

A net $N$ is covered by a set of S-components $\left\{N_{1}, \ldots, N_{n}\right\}$ if every place of $N$ is contained in some $s$ component $N_{i}$ of this set.

THEOREM 5.7. Let $\left(N, M_{0}\right)$ be a live and safe extended free-choice system. Then $N$ is covered by a set of s-components $\left\{N_{1}, \ldots, N_{n}\right\}$ such that each $N_{i}$ has exactly one place marked by $M_{0}$ (this place contains only one token because $\left(N, M_{0}\right)$ is safe).

It is first shown that (i) holds for $s$ systems then using Theorem 5.7 it is proved that it holds for live and safe extended free-choice systems. The meaning of (i) can be illustrated with an example. There exist $M_{1} \stackrel{\sigma}{\longrightarrow} M_{2}$ for the sequence

$$
\sigma=t_{2} t_{4} t_{3} t_{1} t_{2} t_{5} t_{1} t_{2} t_{4} t_{2}
$$

The conflict sets of the net are $\left\{t_{1}\right\},\left\{t_{2}, t_{3}\right\}$, and $\left\{t_{4}, t_{5}\right\}$. The last transition of $\left\{t_{2}, t_{3}\right\}$ occurring in $\sigma$ is $t_{2}$ and the last transition of $\left\{t_{4}, t_{5}\right\}$ occurring in $\sigma$ is $t_{4}$. Therefore, the only conflict order that agrees with $\sigma$ is the one given by $t_{3}<t_{2}$ and $t_{5}<t_{4}$.

Now, (i) asserts the existence of $\leq$-sorted permutation $\tau$ of $\sigma$ such that $M_{1} \stackrel{\tau}{\longrightarrow} M_{2}$, that is, a permutation of $\sigma$ where $t_{3}$ does not occur anymore after the first occurrence of $t_{2}$ and $t_{5}$ does not occur anymore after the first occurrence of $t_{4}$. In this example, the permutation is unique, that is,

$$
\tau=t_{3} t_{1} t_{2} t_{5} t_{1} t_{2} t_{4} t_{2} t_{4} t_{2}
$$

The condition requiring the conflict order to agree with $\sigma$ is essential for the result. In the authors' example, no permutation of $\sigma$ sorted with respect to a conflict order where $t_{2}<t_{3}$ satisfies $M_{1} \stackrel{\tau}{\longrightarrow} M_{2}$, because every nonempty occurrence sequence leading to $M_{2}$ must have $t_{2}$ as the last transition. 
The rest of this section is organized as follows. We prove (i) for $S$-systems. Actually, we prove a stronger result in Proposition 5.10. We prove (i) for live and safe extended free-choice systems in Proposition 5.12. Finally, we obtain the desired upper bound in Theorem 5.13.

5.1. $S$-systems. The result we wish to prove has a strong graph-theoretical flavor, because the occurrence sequences of safe $S$-systems correspond to paths of the $s$-net, as we could observe in the example above. In fact, the main idea of our proof is taken from the proof of the BEST theorem in [9] of graph theory which gives the number of Eulerian trails of a directed graph. In [2, 9], it is cited as the original reference. The following lemma follows immediately from the definitions.

LEMMA 5.8. Let $\left(N, M_{0}\right)$ be an $S$-system and let $M_{1}$ be a reachable marking. Then

$$
\sum_{s \in S} M_{0}(s)=\sum_{s \in S} M_{1}(s)
$$

where $S$ is the set of places of $N$.

LEMMA 5.9. Let $\left(N, M_{0}\right)$ be an $S$-system, let $M_{1}$ be a reachable marking, and let $M_{1} \stackrel{\sigma}{\longrightarrow}$ $M_{2}$ be an occurrence sequence. Let $\leq$ be a conflict order which agrees with $\sigma$ and let $T_{m}$ be the set of maximal transitions (with respect to $\leq$ ).

Let $V_{m} \subseteq T_{m}$ be the set of maximal transitions occurring in $\sigma$. Then every circuit of $N$ containing only transition of $V_{m}$ contains some place marked at $M_{2}$.

Proposition 5.10. Let $\left(N, M_{0}\right)$ be an $S$-system, let $M_{1}$ be a reachable marking, and let $M_{1} \rightarrow M_{3}$ be an occurrence sequence. Let $\leq$ be a conflict order which agrees with $\sigma$. If a prefix of $\leq$-sorted permutation of $\sigma$ is enabled at $M_{1}$, then it can be extended to a $\leq$-sorted permutation of $\sigma$ which is also enabled at $M_{1}$.

Proof. Let $\tau$ be a prefix of a $\leq$-sorted permutation of $\sigma$ such that $M_{1} \stackrel{\tau}{\longrightarrow} M_{3}$ for some marking $M_{3}$. It suffices to prove that if $\tau$ is not a permutation of $\sigma$, then $M_{3}$ enables some transition $t$ such that it is again a prefix of a $\leq$-sorted permutation of $\sigma$. The desired $\leq$-sorted permutation can be constructed by repeatedly extending $\tau$.

Since $N$ is an $s$-net, a transition is enabled if the unique place in its preset is marked. So it suffices to prove that there exists a place $s$, marked at $M_{3}$ such that $\#(s \bullet, \tau)<$ $\#(s \bullet, \sigma)$. Then by Lemma 5.4, the least (with respect to $\leq$ ) transition $t \in s \bullet$ satisfying $\#(t, \tau)<\#(t, \sigma)$ is a feasible extension of $\tau$, that is, $\tau$ is again a prefix of a $\leq$-sorted permutation of $\sigma$.

Assume that no such place $s$ exists, that is, assume that every place $s$ satisfies either

$$
M_{3}(s)=0 \quad \text { or } \quad \#(s \bullet, \tau)=\#(s \bullet, \sigma) .
$$

We first claim that $M_{3}=M_{2}$. By Lemma 5.8, both $M_{2}$ and $M_{3}$ put the same number of tokens in the places of $N$; so it suffices to prove that $M_{2}(s) \geq M_{3}(s)$ for every place $s$. Let $s$ be a place. If $M_{3}(s)>0$, then, by assumption,

$$
\#(s \bullet, \tau)=\#(s \bullet, \sigma)
$$


So

$$
\begin{aligned}
M_{2}(s)= & M_{1}(s)+\#(\bullet s, \tau)-\#(s \bullet, \sigma) \\
& \left(M_{3} \stackrel{\sigma}{\longrightarrow} M_{2}\right) \\
\geq & M_{1}(s)+\#(\bullet s, \tau)-\#(s \bullet, \sigma)
\end{aligned}
$$

( $\tau$ is a prefix of a permutation of $\sigma$ )

$$
=M_{1}(\bullet s)+\#(\bullet s, \tau)-\#(s \bullet, \tau)
$$

(by assumption)

$$
\begin{aligned}
= & M_{3}(s) \\
& \left(M_{1} \stackrel{\tau}{\longrightarrow} M_{3}\right),
\end{aligned}
$$

which finishes the proof of the claim.

Let $T_{m}$ be the maximal transitions with respect to $\leq$. Let $U$ be the set of transitions $t$ satisfying $\#(t, \tau)<\#(t, \sigma)$ and let $U_{m}=U \cap T_{m}$.

Since $\tau$ is not a permutation of $\sigma, u$ is nonempty. We show that $U_{m}$ is also nonempty. Let $t$ be an arbitrary transition in $U$ and let $t_{m}$ be the maximal transition of the conflict set containing $t$. Since $\leq$ agrees with $\sigma$ and $t$ occurs in $\sigma$, the transition $t_{m}$ also occurs in $\sigma$. Since $\tau$ is a prefix of a $\leq$-sorted permutation of $\sigma$ and since $t$ belongs to $U, t_{m}$ belongs to $U$, too. So $t_{m} \in U_{m}$, which implies that $U_{m}$ is nonempty.

We next show that

$$
U_{m}^{\bullet} \subseteq U_{m}
$$

Let $s$ be a place of $U_{m}^{\bullet}$. We prove that $s \in{ }^{\bullet} U_{m}$. Since $M_{2}=M_{3}$, we have

$$
\#(\bullet s, \sigma)-\#(s \bullet, \sigma)=\#(\bullet s, \tau)-\#(s \bullet, \tau)
$$

Since $\tau$ is a prefix of a permutation of $\sigma$, we get

$$
\#(t, \tau) \leq \#(t, \sigma)
$$

for every transition $t$. Moreover, $\#(\bullet s, \tau)<\#(\bullet s, \sigma)$ because $s \in U_{m}^{\bullet}$ and $U_{m}^{\bullet} \subseteq U^{\bullet}$. So $\#(s \bullet, \tau)<\#(s \bullet, \sigma)$, that is, some transition $t \in s \bullet$ belongs to $U$. In particular, $t$ occurs in some transition $t \in s$ that belongs to $u$. In particular, $t$ and the maximal transition in $s \bullet$, say $t_{m}$, both occur in $\sigma$. As $\tau$ is a prefix of a $\leq$-sorted permutation of $\sigma$, we get $t_{m} \in U$. Therefore since $t_{m}$ is maximal, it belongs to $U_{m}$. As $t_{m} \in s \bullet$, we obtain $s \in U_{m}$.

Since $U_{m}$ is nonempty and finite, and by $U_{m}^{\bullet} \subseteq{ }^{\bullet} U_{m}$, there exists a circuit $C$ of $N$ whose transitions belong to $U_{m}$. Let $V_{m}$ be the set of maximal transitions occurring in $\sigma$. We have $U_{m} \subseteq V_{m}$, because all transitions of $U_{m}$ are maximal and occur in $\sigma$. Therefore, 
all the transitions of $c$ belong to $V_{m}$. We can now apply Lemma 5.9 to conclude that $c$ contains some place $s$ marked at $M_{2}$. Since $M_{2}=M_{3}, s$ is also marked at $M_{3}$. Moreover, since $c$ contains only transitions of $U_{m}$, some transition in the post-set of s belongs to $U_{m}$. Since $U_{m} \subseteq U$, this contradicts the assumption that every place marked at $M_{3}$ satisfies

$$
\#(s \bullet, \tau)=\#(s \bullet, \sigma) .
$$

5.2. Extended free-choice systems. Theorem 5.7 suggests looking at extended freechoice systems as a set of sequential systems which communicate by means of shared transitions [14]. The following lemma states that the projection of an occurrence sequence of the systems on one of its $S$-components yields a local occurrence sequence of the component.

LEMMA 5.11. Let $\left(N, M_{0}\right)$ be a system. Let $M_{1}$ be a reachable marking and let $M_{1} \stackrel{\sigma}{\longrightarrow}$ $M_{2}$ be an occurrence sequence. Let $N_{i}$ be an $S$-component of $N$. Let $M_{1}^{i}$ (resp., $M_{2}^{i}$ ) be the restriction of the marking $M_{1}$ (resp., $M_{2}$ ) to the places of $N_{i}$. Let $\sigma_{i}$ denote the sequence obtained from $\sigma$ by deletion of all transitions which do not belong to $N_{i}$; then $M_{1}^{i} \stackrel{\sigma_{i}}{\longrightarrow} M_{2}^{i}$ is an occurrence sequence of $N_{i}$. Using this lemma, it is now shown that Proposition 5.10 also holds for live and safe extended free-choice systems.

Proposition 5.12. Let $\left(N, M_{0}\right)$ be a live and safe extended free-choice system. Let $M_{1}$ be a reachable marking and let $M_{1} \stackrel{\sigma}{\longrightarrow} M_{2}$ be an occurrence sequence. Let $\leq$ be a conflict order which agrees with $\sigma$. If a prefix of $a \leq$-sorted permutation of $\sigma$ is enabled at $M_{1}$, then it can be extended to a s-sorted permutation of $\sigma$ which is also enabled at $M_{1}$.

Proof. By Theorem 5.7, $N$ is covered by a set $\left\{N_{1}, \ldots, N_{n}\right\}$ of $S$-components with exactly one place marked. In the sequel we call these $S$-components state machines of $N_{i}$. Let $N_{i}$ be a state machine of $N$. For each marking of $N$ we define $M^{i}$ as the restriction of $M$ to the set of places of $N_{i}$. For a sequence of transitions $p_{1}, p_{i}$ denotes the sequence obtained from $p$ by the deletion of all transitions which do not belong to $N_{i}$. Let $\tau$ be a proper prefix of a $\leq$-sorted permutation of $\sigma$ such that $M_{1} \stackrel{\tau}{\longrightarrow} M_{3}$ for some making $M_{3}$. Let $U$ be the set of transitions $t$ satisfying \# $(t, \tau)<\#(t, \sigma)$, then $u$ is nonempty. We prove that there exists a transition $t$ of $u$, enabled at $M_{3}$ such that $\tau t$ is again a prefix of a $\leq$-sorted permutation of $\sigma$. As in Proposition 5.10, the desired permutation can then be constructed by repeatedly extending $\tau$.

It suffices to prove that $M_{3}$ enables some transition of $U$. If $u \in U$ is enabled at $M_{3}$, then $N$ is extended free-choice and every transition in the conflict set that contains $u$ is enabled at $M_{3}$. Then by Lemma 5.4, the least (with respect to $\leq$ ) transition $t$ in this conflict set that belongs to $U$ is a feasible extension of $\tau$; that is, $\tau t$ is again a prefix of a $\leq$-sorted permutation of $\sigma$.

We proceed indirectly and assume that no transition of $U$ is enabled at $M_{3}$. Then, since $\left(N, M_{0}\right)$ is a live system and $M_{3}$ is a reachable marking, there exists an $M_{3} \stackrel{p}{\longrightarrow} M$ nonempty occurrence sequence such that $M$ enables some transition $u$ of $U$. We can 
assume that $p$ is a minimal sequence satisfying this property, that is, no intermediate marking enables a transition of $U$.

By assumption, $u$ is not enabled at $M_{3}$, so $M_{3}(s)=0$ for some place $s$ in the preset of $U$. Let $N_{i}$ be a state machine of $N$ containing $s$. By the definition of a state machine and by Lemma 5.8, only one place of $N_{i}$ is marked at $M_{3}^{i}$; let $r$ be this place. By Lemma 5.11, $M_{1}^{i} \stackrel{\sigma_{i}}{\longrightarrow} M_{2}^{i}$ are occurrence sequences of $N_{i}$. Let $\leq_{i}$ be the restriction of $\leq$ to pairs of transition of $N_{i}$. Then $\leq_{i}$ agrees with $\sigma_{i}$ because every conflict set of $N_{i}$ is a conflict set of $N$, by the definition of an $S$-component. Moreover, $\tau_{i}$ is a prefix of a $\leq_{i}$-ordered permutation of $\sigma_{i}$, hence by Proposition 5.10 we have $M_{1}^{i} \stackrel{\tau_{i}}{\longrightarrow} M_{2}^{i}$ for a sequence $\tau^{\prime}$ such that $\tau_{i} \tau_{i}^{\prime}$ is a $\leq$-sorted permutation of $\sigma_{i}$ for every transition of $N_{i}$ that belongs to $U$. Since $u \in U, u$ occurs in $\tau^{\prime} i$. In particular, $\tau_{i}^{\prime}$ is not empty. Let the first $\left(\tau_{i}\right)$ be the first transition of $\tau_{i}^{\prime}$. Since $r$ is the unique place of $N_{i}$ marked at $M_{3}^{i}$, we have the first $\left(\tau_{i}^{\prime}\right) \in r \bullet$. Again by Lemma $5.11 M_{1}^{i} \stackrel{\sigma_{i}}{\longrightarrow} M_{2}^{i}$ and the sequence $p$ contains some transition in $\bullet s$. Since all transitions in $\bullet s$ belong to $N_{i}, p$ contains some transition of $N_{i}$. So $p_{i}$ is nonempty. Let the first $\left(p_{i}\right)$ be the first transition of $p_{i}$. Again, since $r$ is the unique place of $N_{i}$ marked at $M_{3}$, we have the first $\left(p_{i}\right) \in r \bullet$.

So the first $\left(\tau_{i}^{\prime}\right)$ and first $\left(p_{i}\right)$ belong to the same conflict set $r \bullet$. By the extended free-choice property, a marking enables the first $\left(p_{i}\right)$ if and only if it enables the first $\left(\tau^{\prime} i\right)$. Since the first $\left(p_{i}\right)$ occurs in $p$, it becomes enabled after the occurrence of a proper prefix of $p$. So first $\left(\tau_{i}^{\prime}\right)$ becomes enabled after the occurrence of the same proper prefix of $p$ as well. But the first $\left(\tau_{i}^{\prime}\right)$ is a transition of $u$, which contradicts our hypothesis about the sequence $p$. This proves that some transition of $U$ is enabled at $M_{3}$ and we are done.

5.3. An upper bound on the length of shortest paths. We are finally ready to prove the following result.

THEOREM 5.13. Let $\left(N, M_{0}\right)$ be a live and safe extended free-choice system where $N=(S, T, F)$ and let $M_{2}$ be a marking reachable from $M_{1}$. There exists an occurrence sequence $M_{1} \stackrel{\tau}{\longrightarrow} M_{2}$ such that the length of $\tau$ is at most $|T| \cdot|T+1| \cdot|T+2| / 6$.

Proof. Since $M_{2}$ is reachable from $M_{1}$, there exists an occurrence sequence $M_{1} \stackrel{\sigma}{\longrightarrow}$ $M_{2}$. Let $\leq$ be an arbitrary conflict order that agrees with $\sigma$. By Proposition 5.12 (taking the empty sequence for $\tau$ ), there is a $\leq$-sorted permutation $p$ of $\sigma$ such that $M_{1} \stackrel{p}{\longrightarrow} M_{2}$. Let $k$ be the number of distinct transitions, then $k \leq|T|$. We show that there exists an occurrence sequence $M_{1} \stackrel{\tau}{\longrightarrow} M_{2}$ such that the length of $\tau$ is at most $k \cdot(k+1) \cdot(k+2) / 6$.

We proceed by induction on $k$.

BASE. For $k=0$, take $\tau=\varepsilon$.

STEP. For $k>0$, let $p_{1}$ be the maximal prefix of $p$ that contains at most one transition of each conflict set $p_{1}$ is biased. Let $p=p_{1} p_{2}$ and $M_{1} \stackrel{p_{1}}{\longrightarrow} M_{2} \stackrel{p_{2}}{\longrightarrow} M_{3}$. By Theorem 3.4, there is an occurrence sequence $M_{1} \stackrel{\tau_{1}}{\longrightarrow} M_{3}$ such that the length of $\tau_{1}$ is at most $k \cdot(k+1) / 2$. If $M_{3}=M_{2}$, then we are finished, because

$$
\frac{k \cdot(k+1)}{2} \leq \frac{k \cdot(k+1) \cdot(k+2)}{6} .
$$


Now assume that $M_{3} \neq M_{2}$, then $p_{2}$ is nonempty. Let $t$ be its first transition. Since $p_{1}$ is maximal, therefore $p_{1}$ contains a transition $u$ in the conflict set of $t$. Since $p$ is a $\leq$-sorted permutation, $u<t$, and $u$ does not occur in $p_{2}$, so the number of distinct transitions occurring in $p_{2}$ is at most $k-1$. By the induction hypothesis, there exists an occurrence sequence $M_{3} \stackrel{\tau_{2}}{\longrightarrow} M_{2}$ such that the length of $\tau_{2}$ is at most

$$
\frac{k \cdot(k-1) \cdot(k+1)}{6} .
$$

Define $\tau=\tau_{1} \tau_{2}$, then $M_{1} \stackrel{\tau}{\longrightarrow} M_{2}$ and the length of $\tau$ is at most

$$
\frac{k \cdot(k+1)}{2}+\frac{k \cdot(k-1) \cdot(k+1)}{6}=\frac{k \cdot(k+1) \cdot(k+2)}{6} .
$$

6. A family of systems with exponential shortest paths. It can be easily shown that a family of systems for which there exists a family no polynomial upper bound in the length of the shortest paths. All the systems of the family are live and safe. They are even persistent; that is, a transition can only cease to be enabled by its own occurrence. The shortest path is the set $\left\{s_{1}, s_{3}, s_{5}, s_{7}, \ldots, s_{4 n-3}, s_{4 n-1}\right\}$ having increments of exponential nature in the number of transitions of the net. This can be easily proved by showing that in order to reach this marking, transition $t_{2 n-1}$ has to occur at least once and for every $1 \leq i<n$, transition $t_{2 i-1}$ has to occur one time more than twice as often as transition $t_{2 i+1}$.

7. Conclusions. We have obtained polynomial bounds for the length of the shortest paths connection of two given markings for three classes of net systems: safe conflictfree systems, safe marked graphs, and live and safe extended free-choice systems. Furthermore, we have shown that in the case of safe conflict-free systems and safe marked graphs, the bound is reachable and that the length of the shortest paths in safe persistent systems can be exponential in the number of transitions. In the proofs we have made strong use of results of Yen [19] on conflict-free systems and of graph-theoretical results on Eulerian trails. Using the first result (1.1) of this paper, it has been proved that the model checker described there has polynomial complexity in the size of the system for safe conflict-free systems. The third result (1.3) proves that the reachability problem for live and safe extended free-choice systems belongs to the class NP. Although we believe that this problem is solvable in polynomial time, membership in $N P$ is the best upper bound obtained so far. Also, we do not know at the moment if the bound for live and safe free-choice systems is reachable. In fact, we believe that a reachable bound should be quadratic in the number of transitions.

ACKNOWLEDGEMENTs. The author thanks anonymous referees for several helpful comments and suggestions. The author is also thankful to Professor H. C. Pande, ViceChancellor Emeritus, Professor S. K. Mukherjee, Vice-Chancellor, and Professor N. C. Mahanti, Professor and Head of the Department of Applied Mathematics, Birla Institute of Technology, Mesra, for encouragement and support. 


\section{REFERENCES}

[1] T. V. Ardenne-Ehrenfest and N. G. De Bruijn, Circuits and trees in oriented linear graphs, Simon Stevin 28 (1951), 203-217.

[2] E. Best and J. Desel, Partial order behaviour and structure of Petri nets, Formal Aspects Compute. 2 (1990), no. 2, 123-138.

[3] E. Best and J. Esparza, Model checking of persistent Petri nets, 5th Workshop Computer Science Logic, Lecture Notes in Computer Science, vol. 626, Springer-Verlag, Berlin, 1992, pp. 35-52.

[4] E. Best and P. S. Thiagarajan, Some classes of live and safe Petri nets, Concurrency and Nets, Springer, Berlin, 1987, pp. 71-94.

[5] E. Best and K. Voss, Free choice systems have home states, Acta Inform. 21 (1984), no. 1, 89-100.

[6] F. Commoner, A. W. Holt, S. Even, and A. Pnueli, Marked directed graphs, J. Comput. System Sci. 5 (1971), 511-523.

[7] J. Esparza, A solution to the covering problem for 1-bounded conflict-free Petri nets using linear programming, Inform. Process. Lett. 41 (1992), no. 6, 313-319.

[8]___ Model checking using net unfoldings, TAPSOFT '93: Theory and Practice of Software Development (Orsay, 1993), Lecture Notes in Comput. Sci., vol. 668, Springer, Berlin, 1993, pp. 613-628.

[9] H. Fleischner, Eulerian Graphs and Related Topics. Vol. 1, Annals of Discrete Mathematics, vol. 45, North-Holland Publishing, Amsterdam, 1990.

[10] H. J. Genrich and K. Lautenbach, Synchronisationsgraphen, Acta Informat. 2 (1973), 143161 (German).

[11] M. Hack, Analysis of production schemata by petrinets, Tech. Report 94, MIT-MAC, 1972, Corrections, 1974.

[12] R. Howell and L. Rosier, Completeness results for conflict-free vector replacement systems, J. Comput. System Sci. 37 (1988), no. 3, 349-366.

[13] _ Problems concerning fairness and temporal logic for conflict-free Petri nets, Theoret. Comput. Sci. 64 (1989), no. 3, 305-329.

[14] L. H. Landweber and E. L. Robertson, Properties of conflict-free and persistent Petri nets, J. Assoc. Comput. Mach. 25 (1978), no. 3, 352-364.

[15] K. L. Mcmillan, Using unfoldings to avoid the state explosion problem in the verification of asynchronous circuits, 4 th Workshop Computer Science Logic, Springer, London, 1991, pp. 164-174.

[16] P. K. Mishra, A study of the data structure and algorithm analysis in C-programming language, M. Phil, APS University, Rewa India, 1995.

[17] P. K. Mishra and C. K. Sharma, A computational study of an efficient shortest path algorithms in C-programming language, 5 th International Conference on Application of HPC in Engineering, WIT Press, Spain, 1997, pp. 85-90.

[18] P. S. Thiagarajan and K. Voss, A fresh look at free choice nets, Inform. and Control 61 (1984), no. 2, 85-113.

[19] H. C. Yen, A polynomial time algorithm to decide pairwise concurrency of transitions for 1-bounded conflict-free Petri nets, Inform. Process. Lett. 38 (1991), no. 2, 71-76.

[20] H. C. Yen, B. Y. Wang, and M. S. Yang, A unified approach for reasoning about conflict-free Petrinets, Application and Theory of Petri Nets 1993, Lecture Notes in Comput. Sci., vol. 691, Springer, Berlin, 1993, pp. 513-531.

P. K. Mishra: Department of Applied Mathematics, Birla Institute of Technology, Mesra 835215, Ranchi, India

E-mail address: pkmishra@bitmesra.ac.in; pkmishra@ieee.org 


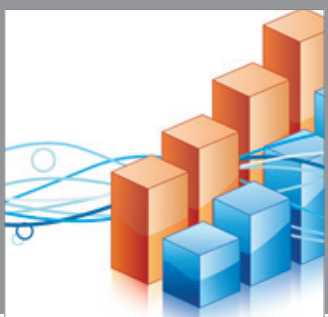

Advances in

Operations Research

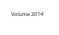

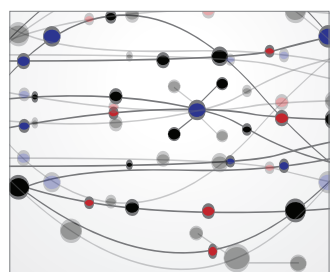

\section{The Scientific} World Journal
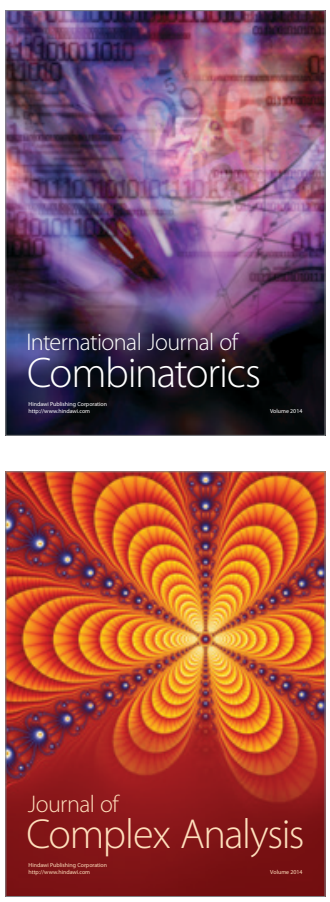

International Journal of

Mathematics and

Mathematical

Sciences
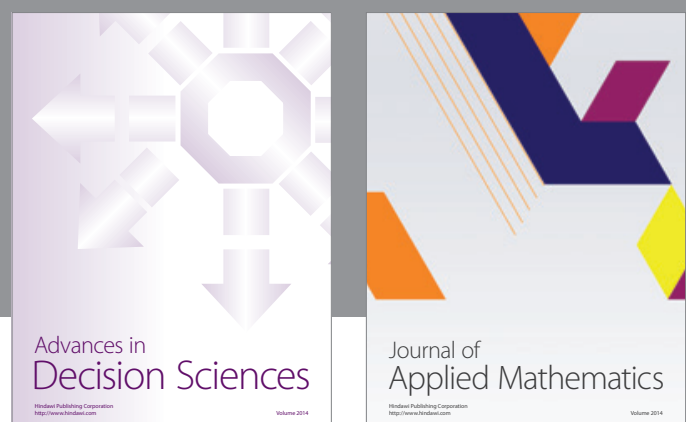

Journal of

Applied Mathematics
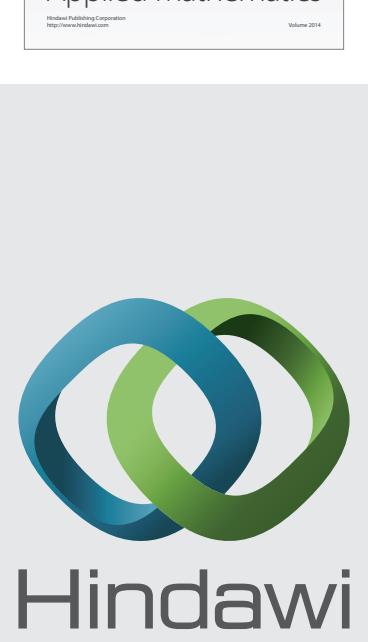

Submit your manuscripts at http://www.hindawi.com
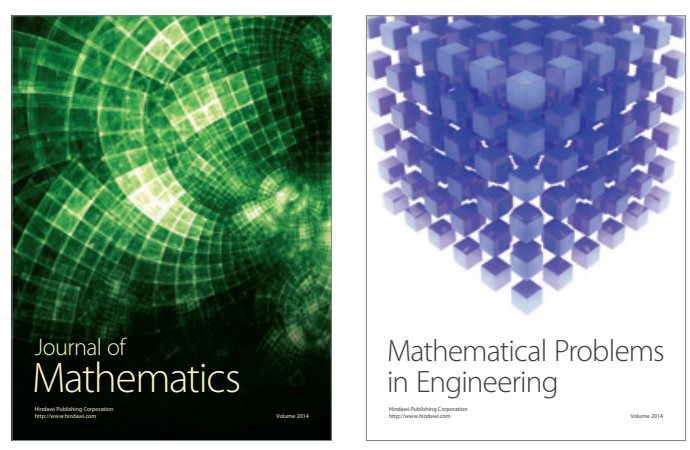

Mathematical Problems in Engineering
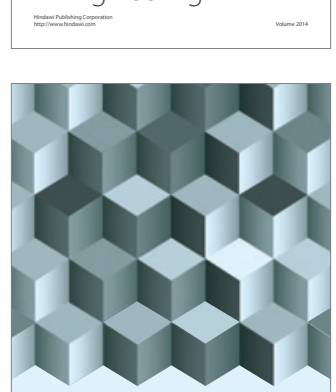

Journal of

Function Spaces
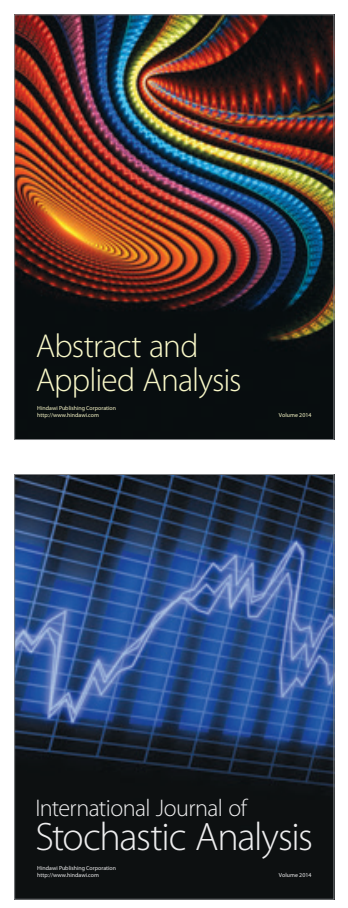

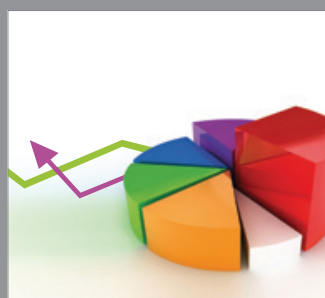

ournal of

Probability and Statistics

Promensencen
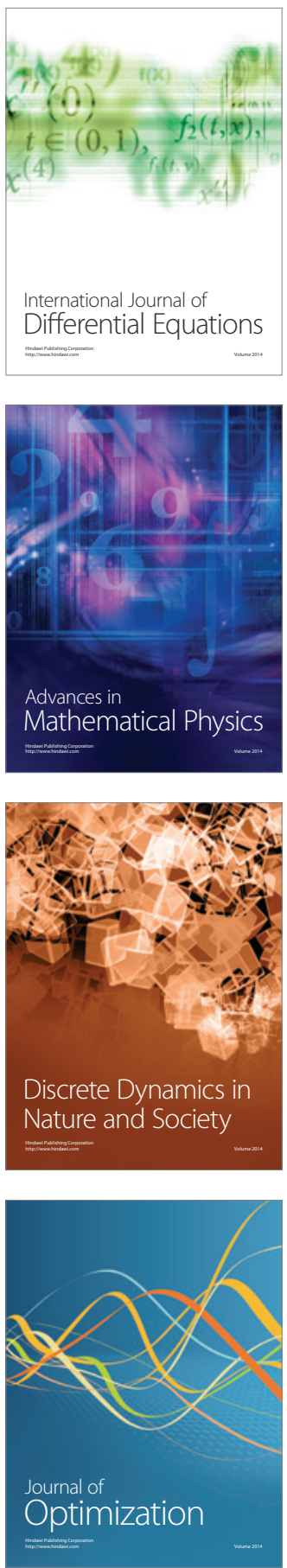